\title{
Water Jet Guided Laser Cutting of High Temperature Superconductors
}

\author{
Eva-Maria Heilmann ${ }^{*}$, Thomas Kowalski ${ }^{*}$, J. Teigelkötter ${ }^{*}$, and Ralf Hellmann ${ }^{*}$ \\ *University of Applied Sciences Aschaffenburg, Wuerzburger Strasse 45, D-63743 Aschaffenburg \\ E-mail: ralf.hellmann@h-ab.de
}

\begin{abstract}
In order to reduce their hysteresis losses, water jet guided laser cutting is applied to process $55 \mu \mathrm{m}$ thin high temperature superconducting tapes. A $100 \mathrm{~W}$ fiber coupled $532 \mathrm{~nm}$ pulsed diode pumped solid state laser is employed in conjunction with a water jet guided cutting system to minimize the burr height and the heat affected zone along the cut kerf. To optimize the laser process a Design of Experiment approach is chosen with the input parameters being laser power, laser repetition rate, tape velocity and water pressure, respectively. To evaluate the improvement of the laser cutting process on the performance of the high temperature superconducting tapes in the $\mathrm{AC}$ modus, hysteresis losses of untreated and laser cut superconducting tapes are compared. As a result, we achieve burr free and clean cut kerfs that lead to critical current losses of $16 \%$ and hysteresis losses on the order of $48 \%$ to $70 \%$, respectively.
\end{abstract}

DOI:10.2961/jlmn.2013.01.0014

Keywords: water jet guided laser cutting, high temperature superconductor, YBCO, current load capacity, hysteresis losses, Design of Experiments

\section{Introduction}

Superconductors are already used in various technical applications, such as DC or AC cables, current limiters or electrical motors [1-3]. Especially, high temperature superconductors (HTS) of the $2^{\text {nd }}$ generation attract considerable interest, among which Yttrium Barium Copper Oxide (YBCO) in form of a coated conductor is one of the most promising materials and architectures characterized by high critical temperatures and high current load capacities in magnetic fields up to several tesla at $77 \mathrm{~K}$ [4]. With the improvement of the production processes of coated conductors their performance nowadays exceeds that of low temperature superconductors and high temperature superconductors of the $1^{\text {st }}$ generation. Furthermore, HTS coated conductors can be manufactured as long tapes with low weight and high mechanical strength [5].

In recent years, the efficiency of such HTS tapes in DC operation was significantly increased [6]. Yet, operation in AC modus shows high hysteresis losses during the exposure of an alternating magnetic field [7-10]. In order to minimize these hysteresis losses, different techniques are employed to sub-divide the conductors into individual segments (linear filaments) [11, 12]. However, chemical or mechanical techniques, such as wet or dry etching as well as mechanical abrasion reduce or even extinguish the superconducting properties of the tapes. In turn, laser material processing allows non-contact, fast and specific processing without mechanical stress and chemical operations. Therefore, various laser systems have been employed for laser ablation and structuring of HTS coated conductors [13, 14].

In an early work, Atanasov et al. investigated structuring YBCO high temperature superconductors using different laser systems, as, e.g., Excimer $\mathrm{XeCl}, \mathrm{CO}_{2}$ and $\mathrm{Nd}$ :YAG, showing that the use of lasers circumvents morphological and compositional changes of the material as it is observed when structuring HTS with conventional processes as photolithography and wet etching [12]. Cobb et al. patterned YBCO thin films into linear striations by using a YAG laser and report a reduction of the hysteresis losses that scales with the filament width in the range between about $100 \mu \mathrm{m}$ and $300 \mu \mathrm{m}$ with the hysteresis losses being lowest at smaller widths [11]. However, the positive effects on the hysteresis losses are hampered by a redeposition of YBCO along the edges of the individual filaments due to the laser ablation process. In this contribution, we report on an alternative approach to reduce the hysteresis losses of YBCO coated conductors while maintaining a high quality laser cut.

We employ the water jet guided laser cutting technique $\left(\right.$ Synova Microjet ${ }^{\mathbb{B}}$ ) rather than scribing only the HTS layer by ablation. Water jet laser cutting is associated with a reduced heat affected zone (HAZ) due to the cooling of the interaction zone by the continuous water jet in between the laser pulses and an efficient removal of the molten material by the water jet. The latter leads to a reduced surface contamination by debris. In addition, a thin water film on the surface prevents resolidification of material by keeping a thin water film on the surface that can be easily cleaned. As a result of these advantages, it has already been shown that water jet guided laser cutting leads to superior cutting results when processing sensitive materials $[15,16]$. Consequently, it can be expected that water jet guided laser cutting will also lead to superior results when processing HTS. In addition, this technology is associated to further advantages, as, e.g., a large working distance due to the stable laminar water jet. This means no further focus control that is typically needed in dry lasers processes ensuring the relative position of the focal point to the work piece is required. This potential of the water jet guided laser has been expounded for different materials, e.g. cutting of silicon, stainless steel sheets or medical stents [17-20].

In our experimental study, we firstly optimized the quality of the laser process cutting HTS tapes with respect to burr formation along the cut kerf, reducing the risk of bridging especially in case of wounding superconducting coils with superimposed tapes. In addition, burr formation 
may hampers the translation in a in a reel-to-reel process and may damages the coated conductor. The optimization is based on a Design of Experiment (DoE) approach, taking into account the most influencing parameters and interactions. Secondly, the current load capacity and hysteresis losses are measured and compared between laser cut and untreated HTS.

\section{Experimental}

\subsection{Superconductor material}

We processed commercially available superconducting tapes with a thickness of about $55 \mu \mathrm{m}$. The architecture of the $3 \mathrm{~mm}$ wide conductor is shown in Figure 1 and consists of a $50 \mu \mathrm{m}$ thick stainless steel substrate, a thin ceramic buffer layer that acts as a template translating the crystal structure to the HTS, the superconductive layer and a silver protection layer, respectively. The HTS layer has been produced by pulsed laser deposition [21]. To maintain a sufficient quality of the conductor, the structure is copper coated after the laser cutting process $(20 \mu \mathrm{m}$ on each side).

\begin{tabular}{|c|l}
\hline $2 \mu \mathrm{m}$ & protection layer \\
$1 \mu \mathrm{m}$ & HTS layer \\
$0.2 \mu \mathrm{m}$ & buffer layer \\
$50 \mu \mathrm{m}$ & substrate \\
\hline $1.8 \mu \mathrm{m}$ & protection layer
\end{tabular}

Fig. 1 Architecture of $2 \mathrm{G}$ high temperature superconductors

\subsection{Laser cutting process}

Due to the low absorption in water in the visible spectral range, our water jet guided laser system is equipped with a fiber coupled $532 \mathrm{~nm}$ pulsed diode pumped solid state laser with an average output power of $100 \mathrm{~W}$ at $40 \mathrm{kHz}$ (Lee Laser LDP-200-MGQ). Power measurements in the water jet guided laser system reveal fiber losses of $4 \%$ and losses at the optical head of $12 \%$. Thus, $84 \%$ of the nominal output power is available for the cutting process.

As HTS coated conductors are manufactured as long tapes (up to several hundred meters), it is desirable to cut such tapes in a continuous process. Hence, the dimensions of a standard 2D laser cutting machine, that typically is constructed to cut material sheets with dimensions ranging from centimeters up to several meters, are not sufficient to process the HTS tapes in an industrial environment. We therefore developed a direct driven coiling device that allows a translation from reel-to-reel and integrated it into the laser cutting machine. Figure 2 shows the coiling device consisting of a profile rail, two coils and two direct driven electric motors. The coils are attached to the motors by an interference fit. To reduce tape vibrations and thus to guarantee a stable cutting process a mechanical support guides the tape in the interaction zone with the water jet and laser. In the process the coiling device is driven by a relative positioning mode or by jog-mode. As only one motor winds up the tape in the reel-to-reel process, the second motor serves as a brake and keeps it taut. The velocity of the coiling device can be varied between 5 and 1200 $\mathrm{mm} / \mathrm{s}$.

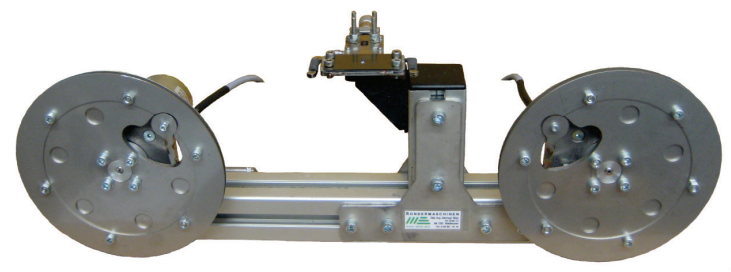

Fig. 2 Coiling Device for a reel-to-reel laser cutting process of superconducting tapes

For cutting multi-filaments (in our case a threefold filament is cut) in a reel-to-reel process, the laser is positioned stationary above the running tape. The orientation of the tape in the coiling device is as shown in Figure 1 with the protection layer being upside. Once the tape has reached its process velocity, the length of the cutting process is controlled by the laser on time. To realize several cuts, the laser is translated perpendicular to the laser cut and the tape is translated backwards. The burr formation on the bottom side of the HTS tape as exemplarily shown in Figure 3 is measured by either a micrometer gauge or a digital microscope (Leica DM6000M).

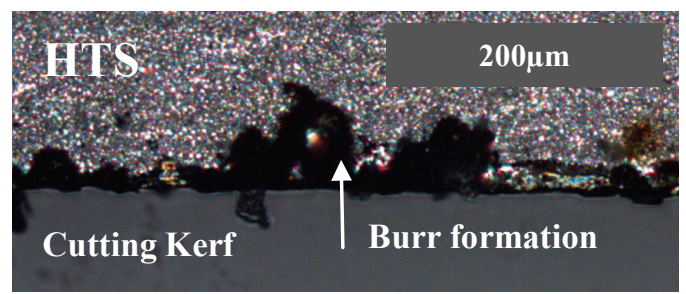

Fig. 3 Microscope picture of the burr formation along the cutting kerf (to illustrate the dimensions the inset bar represents $200 \mu \mathrm{m}$ )

\subsection{Current load capacity and hysteresis losses}

The current load capacity in our study is measured for $20 \mathrm{~cm}$ long tapes in DC mode at liquid nitrogen temperature. In this mode the current is increased until a voltage drop is observed. This quenching characterizes the critical current $\mathrm{I}_{\mathrm{c}}$. The measurement of the hysteresis losses is carried out exposing the superconducting tape to an external alternating magnetic field. Two pick-up coils and two field coils allow adjusting the magnetization curve without probation. Subsequently, the superconducting tape $(20 \mathrm{~cm})$ is placed between these coils with the magnetic field being perpendicular on the surface of the HTS tape. The measurement is examined again and the hysteresis losses can be determined by calculating the area below the magnetization curve. Measurements have been carried out at frequencies between 45 and $100 \mathrm{~Hz}$. Since the losses show a linear dependence of the frequency, any test-frequency can be selected. A detailed description of this measurement technique is reported in [22].

\section{Results and Discussion}

\subsection{Laser material processing}

To get acquainted with the process and to identify feasible input parameters first experiments are carried out in different laser power ranges between 17 and 69W. After these tests, it was decided that the following parameters shall be fixed: Nozzle diameter of $80 \mu \mathrm{m}$, pulse repetition 
rate at $40 \mathrm{kHz}$, laser output power of $17 \mathrm{~W}$, tape velocity between 5 and $20 \mathrm{~mm} / \mathrm{s}$ and a water pressures from 50 up to 150bar.

\subsubsection{Full factorial test plans}

Further experiments include different tape velocities and water pressures (full factorial test plans) for the three laser power levels of $17 \mathrm{~W}, 51 \mathrm{~W}$ and $68 \mathrm{~W}$, respectively. Every test plan encompasses five experiments taking into account each parameter combination. In general, these experiments reveal that the HTS is very sensitive to higher laser powers resulting in heat affected zones that cannot be avoided at water pressures below 150bar. As a consequence, the laser power is limited to $17 \mathrm{~W}$. Based on these predefined parameter ranges, the further optimization of the laser cutting process targets to achieve a high cutting quality without any burr formation. To achieve this, the tape velocity and water pressure are varied at a constant laser power of $17 \mathrm{~W}$.

\subsubsection{Optimized parameter set}

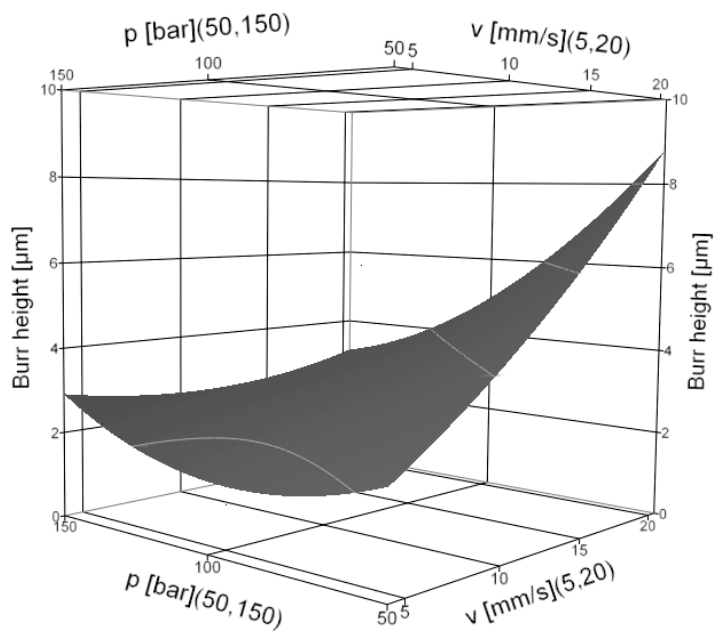

Fig. 4 3-D diagram of the burr height versus water pressure $p$ and tape velocity $\mathrm{v}$ (pre-set laser output power of $17 \mathrm{~W}$ and repetition rate of $40 \mathrm{kHz}$ ).

In order to achieve the optimized parameter combination, the test plan (17W laser output power) is extended by five further experiments. These are necessary to identify linear or quadratic dependencies between the water pressure and the tape velocity. Figure 4 shows the 3-D diagram of the burr height versus water pressure and tape velocity (repetition rate $40 \mathrm{kHz}$, laser output power $17 \mathrm{~W}$ ). In general, 3Ddiagrams serve to illustrate the desired response (in our case the burr height) as a function of two different parameters. All remaining parameters are kept constant. Thus, it is possible to identify the optimized parameter combination. As shown in Figure 4, the tape velocity has a stronger influence on the burr formation as the water pressure with increasing burr height at higher velocities. Therefore, it is preferable to laser cut the HTS tape at lower tape velocities. Every line of the following contour diagram (Figure 5) reflects a cut through the surface of the 3-D diagram. The shaded area describes the preferable burr height of " 0 ". The contour lines are along the resulting burr height of 1, 2, 3, 5 and $7 \mu \mathrm{m}$, respectively, as marked in the diagram.

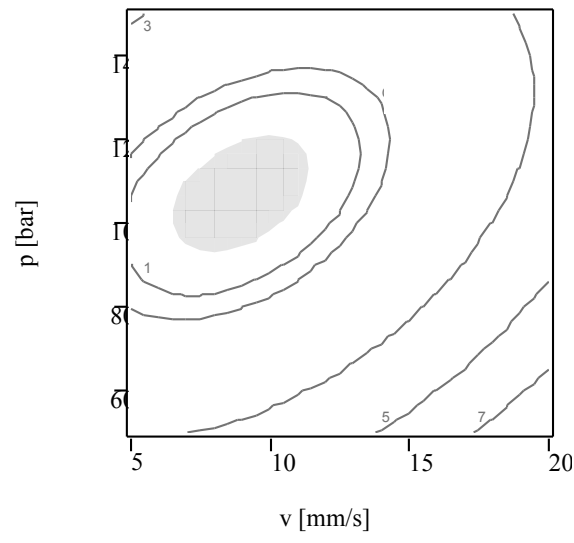

Fig. 5 Contour diagram of the burr height as a function of water pressure $\mathrm{p}$ and tape velocity $\mathrm{v}$. The preferable burr height area of " 0 " is highlighted in grey.

Concluding the results of the laser material process, lowest burr formation can be observed using medium water pressures between 95 and 120 bar in conjunction with low tape velocities between 5 and $10 \mathrm{~mm} / \mathrm{s}$. The optimized parameter combination shown in Table 1 leads to a burr free cutting kerf as demonstrated in Figure 6 (kerf width $=35 \mu \mathrm{m})$. It is worthwhile to mention that as a result of the water jet guided laser cutting process the surface of the tape remains clean and no re-solidified material can be observed.

Table 1 Optimized parameter set

\begin{tabular}{lll}
\hline Parameter & Range & Unit \\
\hline Power & 17 & $\mathrm{~W}$ \\
Tape velocity & 10 & $\mathrm{~mm} / \mathrm{s}$ \\
Water pressure & 100 & $\mathrm{bar}$ \\
Repetition rate & 40 & $\mathrm{kHz}$ \\
\hline
\end{tabular}

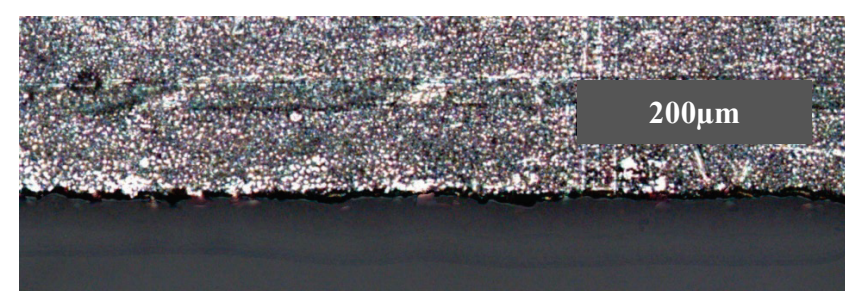

Fig. 6 High quality cutting result using the optimized parameter set

\subsection{Current load capacity and hysteresis losses}

3.2.1 Current load capacity

To analyse and compare the critical current $\mathrm{I}_{\mathrm{c}}$ of the untreated and the laser cut HTS, the measurement setup of chapter 2.3 is used. The results show a current degradation of $17.5 \%$ from $103 \mathrm{~A}$ for the untreated tape and $85 \mathrm{~A}$ for the laser cut tape. This result is consistent with Zhang et al. who report on a critical current loss of $16 \%$ using a standard four-probe measurement method for HTS structured by laser scribing [5]. Since the cutting kerf is only $35 \mu \mathrm{m}$ wide, the geometrically related losses account for $2.5 \%$ and thus the heat input produces $15 \%$ of the current load capacity reduction. Investigations of the individual layers of the 
HTS architecture used in this study reveal numerous cracks on the surface of the buffer layer caused by the laser treatment. As this negatively influences the transfer of the crystal orientation to the HTS layer, $I_{c}$ is reduced [21]. We therefore have to conclude that the employment of the water jet guided laser cutting produces no significant improvement for the critical current but yields comparable results to other structuring techniques.

\subsubsection{Hysteresis losses}

The hysteresis loss can be investigated by positioning linear superconducting tapes between two pick up coils and therefore measuring the magnetization curve. Figure 7 illustrates the hysteresis losses of a laser cut as well as an untreated HTS versus the external magnetic field between 5 and $50 \mathrm{mT}$.

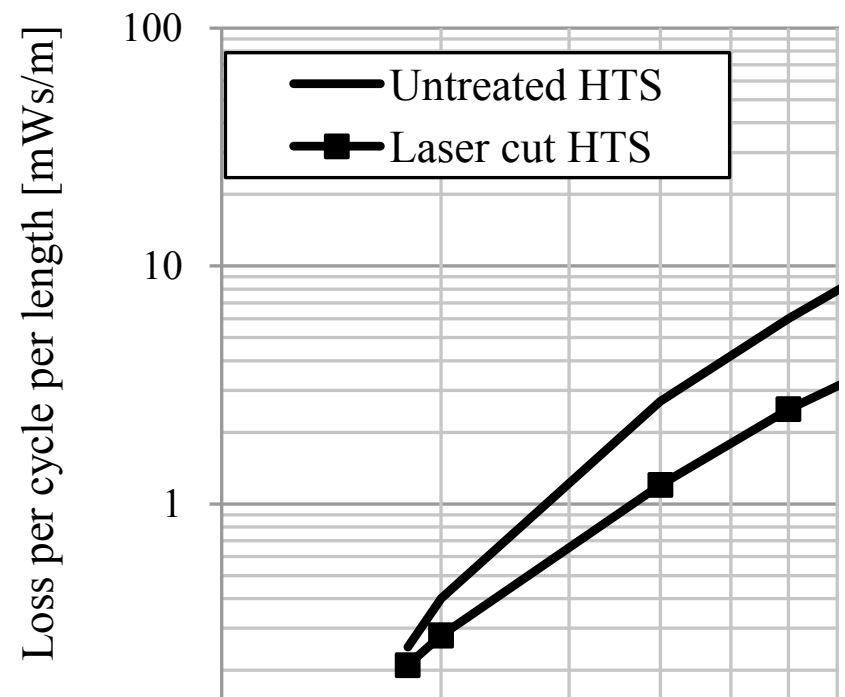

Fig. 7 Hysteresis losses of untreated and laser cut superconductors. As a result of laser material processing, an averaged loss reduction of $48 \%$ can be observed.

Within the illustration we are taking into account the losses per cycle and per length of the conductor, i.e. dividing the measured hysteresis loss by the frequency of the AC magnetic field and the total length of the tape. This evaluation can be carried out due to the linear dependence of the hysteresis losses of the frequency. Figure 7 exhibits the typical trend of increasing losses with increasing magnetic field being in a comparable range as previously reported for other coated conductors [5]. Depending on the details of the architecture and the thickness of the individual layers, we identify an average loss reduction between $48 \%$ and $70 \%$ from untreated to laser cut superconductors. These values correspond well to previously reported loss reductions between $20 \%$ and $91 \%$ which have been achieved for HTS tapes that had been structured by laser scribing [5-7].

\section{Summary}

In summary, we have investigated water jet guided laser cutting of high temperature superconductors employing a fiber coupled $532 \mathrm{~nm}$ pulsed diode pumped solid state laser. Using a DOE approach we identified an optimized parame- ter set, which allows burr free and clean cut kerfs. This high quality cutting result is advantageous for the production process of coated conductors. Furthermore, we report on measuring the current load capacity and the hysteresis losses of the untreated and laser cut material. Measuring the critical current yields losses of $16 \%$, which are comparable to other structuring techniques. The reduction of the hysteresis losses is on the order of $48 \%$ to $70 \%$, being assimilable to other laser based scribing techniques of the HTS layer.

\section{References}

[1] P. Barnes, G. Levin and E. Durkin: Superconducting Generators for Airborne Applications and YBCO Coated Conductors. In: Power and Energy Society General Meeting - Conversion and Delivery of Electrical Energy in the $21^{\text {st }}$ Century, IEEE. Pittsburgh, USA (2008).

[2] M. Gouge, J. Demko, R. Duckworth, D. Lindsay, C. Rey, M. Roden and J. Tolbert: Testing of an HTS Power Cable Made From YBCO Tapes. In: IEEE Transactions on Applied Superconductivity. Vol. 17 (2), 1708-1711 (2007).

[3] M. Iwakuma, Y. Hase, T. Satou, A. Tomioka, M. Konno, Y. Iijima, T. Saitoh, Y. Yamada, T. Izumi and Y. Shiohara: Development of a $7.5 \mathrm{~kW}$ YBCO Superconducting Synchronous Motor. In: IEEE Transactions on Applied Superconductivity. Vol. 18 (2), 689-692 (2008).

[4] C. Freyhardt, J. Hoffmann, J. Wiesmann, J. Dzick, K. Heinemann, A. Isaev, F. Garcia-Moreno, S. Sievers and A. Usoskin: YBaCuO Thick Films on Planar and Curved Technical Substrates. In: IEEE Transactions on Applied Superconductivity. Vol. 7 (2), 1426-1431 (1997).

[5] D. Hazelton and V. Selvamanickam: SuperPower's YBCO Coated High-Temperature Superconducting (HTS)Wire and Magnet Applications. In: Proceedings of the IEEE. Vol. 97 (11), 1831-1836 (2009).

[6] Y. Zhang, R. Duckworth, T. Ha, F. ListIII, M. Gouge, Y. Chen, X. Xiong, V. Selvamanickam and A. Polyanskii: AC Loss Reduction in Filamentized YBCO Coated Conductors With Virtual Transverse Cross-Cuts. In: IEEE Transactions on Applied Superconductivity. Vol. 21(3), 3301-3306 (2010).

[7] N. Amemiya, S. Kasai, K. Yoda, Z. Jiang, G. Levin, P. Barnes and C. Oberly: AC loss reduction of YBCO coated conductors by multifilamentary structure. In: Superconductor Science and Technology. Vol. 17, 1464-1471 (2004).

[8] K. Suzuki, J. Matsuda, M. Yoshizumi, T. Izumi, Y. Shiohara, M. Iwakuma, A. Ibi, S. Miyata and Y. Yamada: Development of a laser scribing process coated conductors for the reduction of AC losses. In: Superconductor Science and Technology. Vol. 20, 822-826 (2007).

[9] J. Ogawa, H. Nakayama, S. Odaka and O. Tsukamoto: AC loss characteristics of YBCO conductors carrying transport currents in external AC magnetic fields. In: Cryogenics. Vol. 45 (1), 23-27 (2003).

[10] M. Iwakuma, M. Nigo, D. Inoue, T. Kiss, K. Funaki, Y. Iijima, T. Saitoh, Y. Yamada and Y. Shiohara: AC 
Loss Properties of YBCO Superconducting Tapes Exposed to External AC Magnetic Field. In: EEE Transaction on Applied Superconductivity. Vol. 15(2), 1562-1565 (2005).

[11]C. Cobb, P. Barnes, T. Haugan, J. Tolliver, E. Lee, M. Sumption, E. Collings, C. Oberly: Hysteretic loss reduction in striated YBCO. In: Physica C. Vol. 382, 5256 (2002).

[12] O. Tsukamoto, N. Sekine, M. Ciszek and J. Ogawa: A Method to Reduce Magnetization Losses in Assembled Conductors Made of YBCO Coated Conductors. In: IEEE Transactions on Applied Superconductivity. Vol. 15 (2), 2823-2826 (2005).

[13]P. Atanasov, R. Tomov, V. Serbesov, A. Grunchev and L. Avramov: Laser patterning and modification of thin YBCO films. In: Proceedings of the SPIE. Vol. 2777, 163-173 (1996).

[14]G. Levin, P. Barnes, and J. Kell: Multifilament $\mathrm{YBa}_{2} \mathrm{Cu}_{3} \mathrm{O}_{6+\mathrm{x}}$-coated conductors with minimized coupling losses. In: Applied Physics Letters. Vol. 89 (1), 1-3 (2006).

[15]B. Richerzhagen, G. Delacrétaz, and R.P. Salathé: Complete Model to simulate the thermal defocussing of a laser beam focused in water. In: Optical Engineering, Vol. 35 (7), 2058-2066 (1996).

[16] A. Richter: Guided by water. In: Cutting Tool Engineering 8, 168 (2004).

[17] N. Muhammad, D. Whitehead, A. Boor and L. Li: Comparison of dry and wet fibre laser profile cutting of thin 316L stainless steel tubes for medical device applications. In: Journal of Materials Processing Technology. Vol 210, 2261-2267 (2010).

[18] A. Pauchard: Precise thin metal cutting using the Laser MicroJet. In: Laser in electronics manufacturing, Proc. of the LEF Conf. Fürth, Germany (2009).

[19]E.M. Heilmann and R. Hellmann: Water Jet Guided Laser Cutting of Silicon Thin Films Using 515nm Disk Laser. In: JLMN. Vol. 6 (2), 168-173 (2011).

[20]F. Wagner, A. Spiegel, and B. Richerzhagen: Water jet guided laser cutting using frequency doubled YAG lasers. In: Laser in electronics manufacturing, Proc. of the LEF Conf. Erlangen, Germany (2004).

[21] J. Greer: High quality YBCO films grown over large areas by pulsed laser deposition. In: Journal of Vacuum Science \& Technology A: Vacuum, Surfaces, and Films. Vol. 10 (4), 1821 - 1826 (1992).

[22] L. Fisher, A. Kalinov and I. Voloshin: Simple Calibration Free Method to Measure AC Magnetic Moment and Losses. In: IEEE/CSC \& ESAS European Superconductivity News Forum. Vol. 3, 1-6 (2008).

(Received: June 28, 2012, Accepted: January 11, 2013) 